\title{
Theory of magnon diffuse scattering in scanning transmission electron microscopy
}

\author{
Keenan Lyon, ${ }^{1, *}$ Anders Bergman, ${ }^{1}$ Paul Zeiger $\odot,{ }^{1}$ Demie Kepaptsoglou $\odot,{ }^{2,3}$ \\ Quentin M. Ramasse $\odot,{ }^{2,4,5}$ Juan Carlos Idrobo, ${ }^{6}$ and Ján Rusz ${ }^{1, \dagger}$ \\ ${ }^{1}$ Department of Physics and Astronomy, Uppsala University, Lägerhyddsvägen 1, Uppsala, Sweden \\ ${ }^{2}$ SuperSTEM Laboratory, SciTech Daresbury Campus, Daresbury WA4 4AD, United Kingdom \\ ${ }^{3}$ Department of Physics, University of York, York YO10 5DD, United Kingdom \\ ${ }^{4}$ School of Chemical and Process Engineering, University of Leeds, Leeds LS2 9JT, United Kingdom \\ ${ }^{5}$ School of Physics and Astronomy, University of Leeds, Leeds LS2 9JT, United Kingdom \\ ${ }^{6}$ Center for Nanophase Materials Sciences, Oak Ridge National Laboratory, Oak Ridge, Tennessee 37831, USA
}

(Received 11 May 2021; revised 30 November 2021; accepted 2 December 2021; published 13 December 2021)

\begin{abstract}
We present a theory and a simulation of diffuse scattering due to the excitation of magnons in scanning transmission electron microscopy. The calculations indicate that magnons can present atomic contrast when detected by electron energy-loss spectroscopy using atomic-size electron beams. The results presented here indicate that the intensity of the magnon diffuse scattering in bcc iron at $300 \mathrm{~K}$ is 4 orders of magnitude weaker than the intensity of thermal diffuse scattering arising from atomic vibrations.
\end{abstract}

DOI: 10.1103/PhysRevB.104.214418

\section{INTRODUCTION}

Efficient electron beam monochromators have extended the already wide range of experimental techniques available to scanning transmission electron microscopy (STEM) by allowing for the measurement of vibrational spectra [1,2]. Since its first demonstration, vibrational electron energy-loss spectroscopy (EELS) is developing at a swift pace. Several key milestones have been reached, such as the identification of isotope compositions [3], the detection of atomic level contrast in vibrational signals $[4,5]$ and of the spectral signatures of an individual impurity atom [6], and spatial- and angleresolved measurements on a single stacking fault [7]. Such vibrational modes occupy an energy range from zero to tens or few hundreds of $\mathrm{meV}$ in solid state materials. Qualitatively the same energy range is also occupied by energy losses due to excitations of magnons, arising from the collective excitation of the electrons' spin in a lattice.

Magnons represent collective excitations of the magnetic subsystem and semiclassically they can be visualized as a wave of precessing magnetic moments [8]. Among other inelastic scattering experimental techniques [9-11], magnons can be efficiently excited by electron scattering in reflection geometry: spin-polarised EELS (SPEELS) or reflection (REELS) using spin- and non-polarised electron sources,

\footnotetext{
*keenan.lyon@physics.uu.se

†jan.rusz@physics.uu.se
}

Published by the American Physical Society under the terms of the Creative Commons Attribution 4.0 International license. Further distribution of this work must maintain attribution to the author(s) and the published article's title, journal citation, and DOI. Funded by Bibsam. respectively [12-14]. It is therefore expected that, in direct analogy with phonon spectroscopy, the spectroscopic signature of magnons and their dispersion in momentum space should also be accessible within the remit of vibrational STEM-EELS. Due to the scattering cross sections and low penetration depth for low-energy electrons (typically the incident beam energy does not exceed $10 \mathrm{eV}$ ), SPEELS (REELS) measurements are restricted to the detection of spin waves at surfaces or ultrathin films. However, although these techniques allow the probing of magnon excitations and their energy-momentum dispersion with high-energy resolution, localised information stemming, for example, from buried interfaces or point defects, is lost. The promise, therefore, of probing magnons with STEM-EELS at simultaneous high spatial and energy resolution is highly attractive.

It is well known that the interaction of magnetic moments with the electron beam is significantly weaker than its interaction with the Coulomb potential, often by 3 or 4 orders of magnitude $[15,16]$. Since magnons represent time-dependent distortions of the magnetic structure, similarly as phonons represent time-dependent distortions of the crystal structure, one might conclude that the inelastic magnon signal will be of 3 to 4 orders of magnitude weaker than the phonon signal, which would certainly make their detection very challenging. In certain contexts, however, the magnetic effects in the elastic scattering regime can reach relative strengths of up to a few percent [17-19].

Furthermore, the use of direct or hybrid-pixel detectors for spectroscopy applications already offers drastically improved detection dynamic range and low background noise, with signals a mere $10^{-7}$ of the full beam intensity readily detectable within tens of pixels of the recorded signal's maximum [20]. Together with improvements in monochromator and spectrometer design, resulting in increased energy resolutions in particular at lower acceleration voltages $(4.2 \mathrm{meV}$ 
at $30 \mathrm{kV}$ [2]), where the inelastic cross sections are more favorable, signals of weak intensity such as energy losses due to excitation of magnons could be accessible experimentally.

Here we devise a model for the simulation of diffuse scattering due to magnons (MDS). Consequent simulations of the inelastic magnon scattering show an intensity distribution of the MDS in the diffraction plane under experimental conditions studied here. We compare the intensity of magnon scattering with that of phonon scattering. We show that with an atomic size electron beam the inelastic magnon scattering displays atomic level contrast. Finally, we briefly outline the challenges that would need to be overcome for an experimental detection of magnon signals in monochromated STEM-EELS.

\section{THEORY AND COMPUTATIONAL DETAILS}

First, we introduce our theoretical model for the calculation of the MDS. The model is based on an analogy with the socalled quantum excitation of phonon (QEP) model [21], where it was argued that the inelastic signal due to atomic vibrations can be accessed via sampling from all possible atomic displacement configurations. Averaging over intensities (squared amplitudes) of exit wave-functions results in an incoherent (total) scattering intensity. Instead, taking the squared amplitude of the averaged auxiliary exit wave function results in a coherent (elastic) scattering intensity. Following Ref. [22] we show in Appendix A that this approach also extends to magnons. The magnon inelastic scattering signal is then the difference between the incoherent and coherent scattering intensities. Instead of vibrating atoms, we deal here with wiggling magnetic moments. They cause local deviations of the microscopic magnetic fields from their time average. These deviations influence the electron beam propagating through the sample and it is what allows an extraction of magnon scattering intensities.

To realize this formal analogy practically, we need three key components. The first component is a beam propagation method that can take into account the influence of a microscopic magnetic field on the electron beam. Such a method exists and has been described in Refs. [15,17,18]. In works of Edström et al., a Pauli multislice method was introduced, which is a generalization of the classical multislice method [23], deriving a paraxial approximation starting from Pauli's equation rather than Schrödinger's equation.

The second component is a method for generating the microscopic magnetic field $\mathbf{B}(\mathbf{r})$ and microscopic magnetic vector potential $\mathbf{A}(\mathbf{r})$. In Refs. [15,17-19], $\mathbf{A}(\mathbf{r})$ and $\mathbf{B}(\mathbf{r})$ were generated from spin-densities evaluated by calculations within the scalar-relativistic density functional theory framework. However, for large supercells containing many thousands of atoms with varying orientations of magnetic moments, such an approach becomes impractical. For this purpose we have employed a recently developed parametrization of the magnetic fields and vector potentials [24].

The parametrization starts with the microscopic magnetic vector potentials and fields of single atomic systems, where a quasidipole model is fitted for each individual element in a way analogous to electron form factors while also accounting for the reduced magnetic moment in the atomic to bulk transition. As the contributions to these magnetic quantities from each atom can simply be summed up in superposition, knowledge of the positions and moment directions for a supercell in tandem with this parametrization allows for efficient computation of these magnetic quantities in systems of any size.

The third and final component provides realistic snapshots of precessing magnetic moments in the supercell. This can be efficiently realized by semiclassical atomistic spin dynamics (ASD) simulations [8]. ASD is a magnetic analog of how molecular dynamics (MD) simulations describe atomic vibrations, here providing a realistic description of thermally excited magnetic configurations and their dynamics. Here, we have used the UppASD code [25] for the ASD simulations.

The parameter settings for each step are described in reverse order. As our model system we chose ferromagnetic bcc iron, a prototypical system for $a b$ initio calculations of magnetic properties, and one where magnons have been previously detected experimentally by energy loss spectoscopy using SPEELS [13]. A supercell of $30 \times 30 \times 100$ unit cells with dimensions $86 \times 86 \times 287 \AA^{3}$ containing 180000 atoms, has been constructed. The ASD method relies on a parameterized spin Hamiltonian to describe the spin dynamics. Here we have used a Heisenberg Hamiltonian defined by interatomic exchange interactions calculated $a b$ initio using the scalarrelativistic SPRKKR [26] code. With this realistic description of the spin dynamics in bcc Fe, we performed ASD simulations in order to get representative snapshots of the magnetization in the sample with a sampling interval of $1 \mathrm{ps}$. The simulations were performed with a timestep of $0.1 \mathrm{fs}$ and we used a large Gilbert damping parameter of 0.1 in order to minimize the correlation between different snapshots.

In the generation of parametrized fields we utilize a numerical grid of $1500 \times 1500 \times 3000$ grid points spanning the entire supercell, with points within a $6 \times 6 \times 6 \AA^{3}$ cube surrounding each atom evaluated for the magnetic vector potentials and magnetic fields.

The Pauli's-equation-based multislice calculations have been done on the same numerical grid. The cut-off for atomic potentials, using Kirkland's parametrization [27], was set to $4 \AA$. Zero aberrations for the incoming wave have been assumed, and the beam was focused on the entrance surface of the supercell. The mass and electron wavelength in the Pauli multislice equation is relativistically corrected, as in the traditional multislice method [15,23].

\section{RESULTS}

Since the MDS will create nonzero scattered intensity in between Bragg spots, it will overlap with the diffuse scattering stemming from atomic vibrations (TDS). It is therefore important to know the distribution and intensity of the TDS signal in the diffraction plane. We compute the TDS in a similar fashion to the MDS, but instead of snapshots of the time-varying magnetic field, snapshots of the vibrating crystal structure are required. To that end, we simulate the atomic vibrations with the LAMMPS MD code [28,29]. An orthogonal simulation box consisting of $30 \times 30 \times 100$ unit cells of bcc-Fe was considered with a lattice parameter $a=2.859 \AA$, which was determined from the time average of the simulation box dimensions 

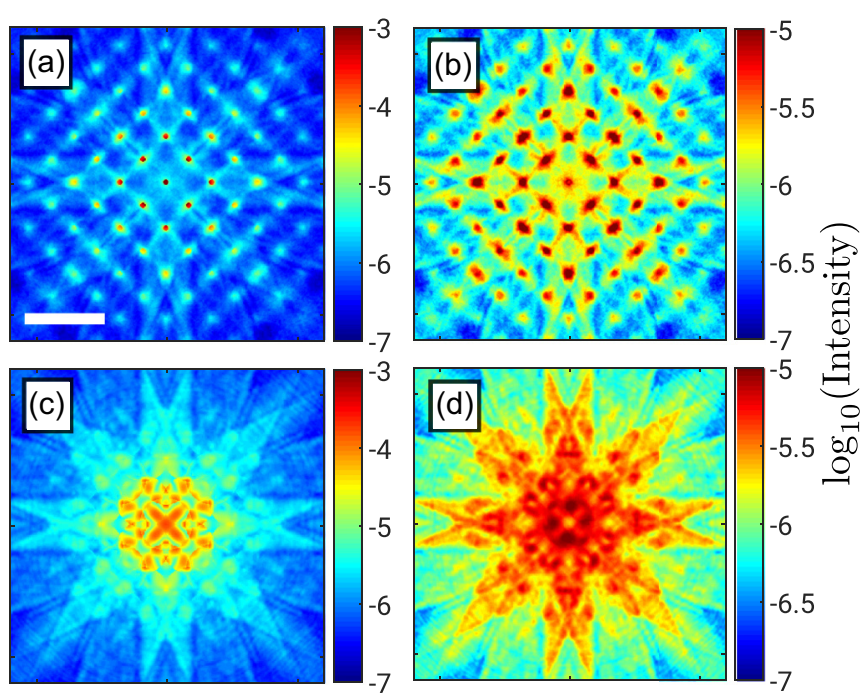

FIG. 1. Thermal diffuse scattering simulation due to atomic vibrations. The acceleration voltage was set to $200 \mathrm{kV}$. This calculation neglects magnetic effects. The convergence semiangle was set to 1 mrad in (a) and (b), and to $10 \mathrm{mrad}$ in (c) and (d), respectively. The total diffraction pattern is shown in panels (a) and (c), while the pure phonon signal is shown in panels (b) and (d). The intensity is plotted on a logarithmic scale for scattering angles of $\pm 60 \mathrm{mrad}$ along both axes, with the white bar in panel (a) representing $30 \mathrm{mrad}$.

via isothermal-isobaric MD simulation at a temperature of $300 \mathrm{~K}$ and a pressure of 0.0 bar. In all MD simulations, the time step was set to $0.001 \mathrm{ps}$ and the embedded-atom method potential described the inter-atomic interactions [30]. One MD trajectory was simulated using a Langevin thermostat at a temperature of $300 \mathrm{~K}$, from which one structure snapshot was taken every 2000 time steps, i.e., every 2 ps, for a total of 96 snapshots. Figure 1 shows a calculated total (incoherent) diffraction pattern and its inelastic phonon component. The relative strength of energy-integrated phonon signal intensity reaches up to $10^{-2}$ of the total intensity.

Recent high-energy-resolution STEM-EELS experiments in the vibrational regime have explored the use of either nanoprobe or atomic resolution modes. Nanoprobe offers nanometer-scale spatial resolution alongside interpretable angle-resolved measurements at moderate momentum resolution [4]. In such a case, small convergence semiangles are being used, such as $1 \mathrm{mrad}$. In atomic resolution mode we form an Ångström-sized electron probe by using substantially larger convergence semiangles, such as $20-30$ mrad. Experiments with atomic size electron beams offer atomic-resolution EELS but without momentum sensitivity [5-7]. Given the practical and conceptual similarities between these vibrational spectroscopy results and the experiments envisaged here to study magnons, these two usecases will form the basis for the choice of simulation parameters.

We start our discussion of MDS with simulations assuming a nanometer-sized electron beam with a convergence semiangle of $1 \mathrm{mrad}$ at an acceleration voltage of $200 \mathrm{kV}$, see Fig. 2. Although lower acceleration voltages, typically 30 $60 \mathrm{kV}$, have been used in recent vibrational and ultralow loss STEM-EELS experiments, due in particular to higher

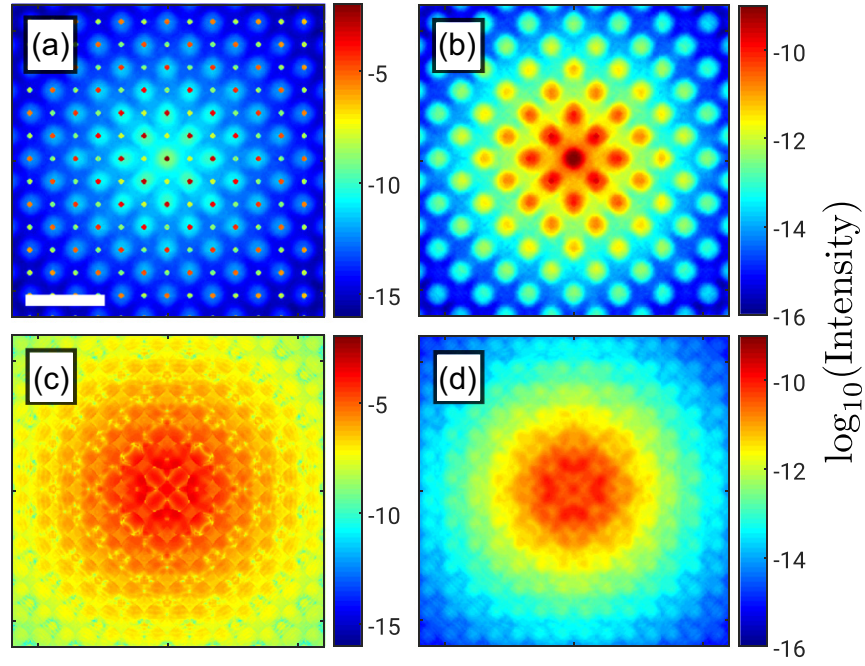

FIG. 2. Diffuse scattering simulation due to precession of magnetic moments, i.e., excitation of magnons. Atomic vibrations are neglected in this simulation. The convergence semiangle was set to 1 mrad in (a) and (b), and to $10 \mathrm{mrad}$ in (c) and (d), respectively. The total diffraction pattern is shown in panels (a) and (c), while pure magnon signal is shown in panels (b) and (d). The intensity is plotted on a logarithmic scale for scattering angles of $\pm 60 \mathrm{mrad}$ along both axes, with the white bar in panel (a) representing $30 \mathrm{mrad}$.

inelastic scattering cross-sections, the sample requirements (e.g., the risk of oxidation in very thin lamellae of bcc-Fe) make the choice of $200 \mathrm{kV}$ pertinent as it would allow for the observation of thicker objects. Figure 2(a) shows the central part of the diffraction pattern within a $\pm 60 \mathrm{mrad}$ range of scattering angles, where the atomic vibrations have been neglected. It can be seen that the intensity of the diffuse signal due to the excitation of magnons is of a similar order of magnitude as forbidden reflections, that is approximately $10^{-6}-10^{-7}$ of the transmitted beam intensity. In a simulation including atomic vibrations the forbidden reflections are not visible, being dominated by the vibrational TDS, see Fig. 1(a). The pure inelastic signal component due to magnons is shown in Fig. 2(b). It forms clouds of intensity centered around the Bragg reflections. The per-pixel maximum relative intensity of the MDS and TDS reaches $3 \times 10^{-4}$. Figures 2(c) and 2(d) show an analogous calculation for an electron beam with convergence semiangle of $10 \mathrm{mrad}$. The relative intensity of the MDS remains at a very similar level. Figures 2(b) and 2(d) represent the energy-integrated magnon EELS signal, i.e., excluding the zero-loss peak and phonon EELS intensity. We have performed similar calculations for a 5-nm thick sample at $30 \mathrm{kV}$ acceleration voltage with qualitatively similar outcomes, see Appendix B.

For convergence semiangles of approximately $10 \mathrm{mrad}$ and beyond, the Bragg discs already overlap, meaning that we are in the atomic resolution regime. An intriguing question arises, whether atomic scale contrast could be reached using purely the magnon EELS signal. In Fig. 3 we present a calculation of a STEM image based purely on the inelastic magnon intensity. A convergence semiangle of $30 \mathrm{mrad}$ and acceleration voltage of $100 \mathrm{kV}$ have been assumed. We have considered three typical detector settings: high-angle annular 


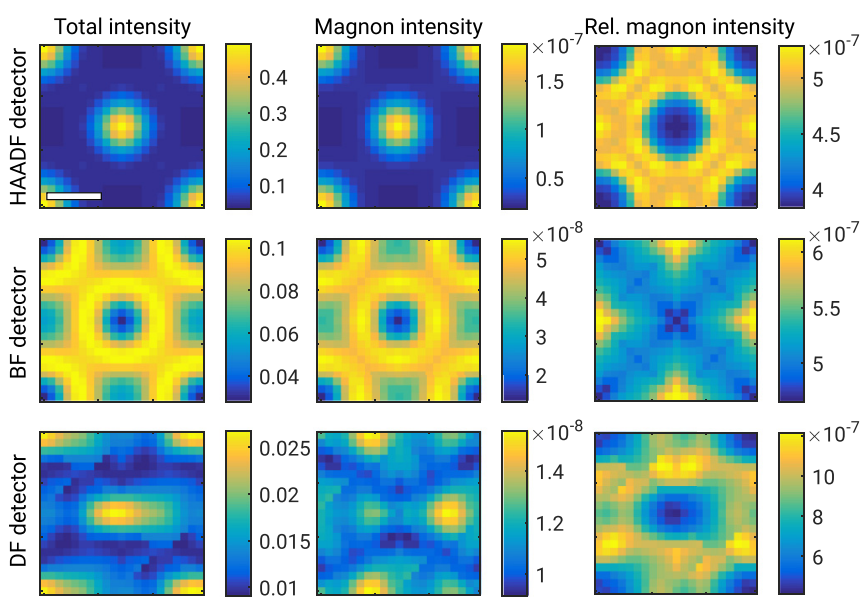

FIG. 3. STEM images calculated with an electron beam accelerated by $100 \mathrm{kV}$ and convergence semiangle of $30 \mathrm{mrad}$, while neglecting the atomic vibrations. The white bar in the top left panel marks $1 \AA$. Individual rows correspond to three different detector setups: HAADF detector with inner/outer collection semiangles of $100 \mathrm{mrad}$ and $250 \mathrm{mrad}$, BF detector with collection semiangle of $30 \mathrm{mrad}$ and a DF detector displaced by $60 \mathrm{mrad}$ off-axis along the $\theta_{x}$ direction, with a collection semiangle of $30 \mathrm{mrad}$. Individual columns correspond to the total intensity (excluding phonon contribution), magnon scattering intensity, and their ratio.

dark field (HAADF) with inner/outer collection semiangles of $100 \mathrm{mrad}$ and $250 \mathrm{mrad}$, bright field (BF) with collection semiangle of $10 \mathrm{mrad}$ and off-axis dark field (DF) detector with collection semiangle of $30 \mathrm{mrad}$, displaced by $60 \mathrm{mrad}$ from the center of diffraction pattern along $\theta_{x}$ axis. These could be thought of as energy-filtered STEM images, that is, spectrum images collected using equivalent collection angle ranges, but generated by integrating the energy range solely over magnon losses. An experimental realization may thus be possible if the most intense magnon peaks are sufficiently separated from other losses in the corresponding energy-loss range.

For all three detector settings we observe an atomic scale contrast, both in total scattering intensity (left column) as well as in the magnon diffuse scattering (middle column). The HAADF detector leads to the highest atomic level contrast, both in total and in magnon scattering intensity. On the other hand, owing to the very similar spatial distribution of both signals, the ratio of the magnon signal intensity to the total scattering intensity (right column) remains under $10^{-6}$. The magnon signal of the BF detector shows expected strong dynamical diffraction effects with volcano-shaped features around the atomic columns. A similar behavior was reported in phonon EELS maps [31]. The contrast is lower with a ratio of maximal to minimal intensities near a factor of 5, while the relative strength of the magnon signal remains below $10^{-6}$, at a similar level to the magnon signal collected by the HAADF detector. For the chosen geometry of the off-axis DF detector, in the total scattering cross section we observe elongated features at the position of atomic columns. Interestingly, the peaks of the magnon signal are found to be strongly displaced to the right, by about $0.8 \AA$ in the direction parallel to the displacement of the detector. Similar observations were made about phonon EELS in Ref. [32], albeit to a smaller extent. Thanks to the qualitatively different STEM image of the total vs magnon signal, the relative strength of magnon scattering intensity is higher here, reaching $1.3 \times 10^{-6}$.

\section{DISCUSSION AND OUTLOOK}

Although this level of signal is small in absolute terms, making their detection highly challenging, we would like to point out some recent instrumentation advances and observations. For instance, the vibrational signature of guanine molecules obtained using a hybrid-pixel detector revealed peaks of intensity well below $2 \times 10^{-5}$ that of the zero-loss peak (ZLP), across an energy-loss range starting as low as $\sim 20 \mathrm{meV}$. [33] Thanks to the increased energy resolution of new-generation monochromators, the numerous peaks in the low energy-loss sector are also well separated, making it easier to isolate the contribution from each allowed vibrational mode into, e.g., an inelastic "phonon" image or "magnon" image, as simulated here. Furthermore, in a dark-field geometry, such as that described above for the HAADF or off-axis DF detectors, the relative intensity of the ZLP is vastly reduced (for a lower overall signal) and its intensity for dark-field phonon imaging is of a similar order of magnitude to that of vibrational peaks [4]. This alternative electron optical geometry should allow for an enhancement of the relative magnon signal in future experiments, especially if direct electron detectors are used.

In the presented simulations it was assumed that the sample is subjected to a $1 \mathrm{~T}$ magnetic field parallel to the beam coming from the objective lens. Newly-developed instrumentation makes it possible to either null or control the magnetic field of the STEM objective lens at the sample while retaining an atomic-size probe [34]. It would thus be of interest to analyze the impact of the strength and direction of the magnetic field on the observed magnon signal as an additional experimental means to boost or isolate magnon peaks in the recorded spectra. Another parameter of great interest is the temperature. It is likely that the temperature dependence of the phonon and magnon signals will qualitatively differ, perhaps even offering routes to attempt the separation of these signals. Bcc iron has a magnetic transition temperature above $1000 \mathrm{~K}$, therefore one would expect stronger magnon scattering intensities at higher than room temperatures. All these aspects will be the subject of future research.

The simulations presented here lack the spectroscopic dimension in the magnon scattering: All calculations are integrated over all possible energy losses where magnons contribute to the spectra. We aim to extend our work to include energy channel sensitivity by analogy with the frequency-resolved frozen phonon multislice method (FRFPMS; $[35,36])$. For that purpose, we are implementing colored thermostats into UppASD. Such simulations will provide snapshots of magnetic moments vibrating only within desired frequency ranges. This will allow us to assemble magnon EEL spectra by repeating the simulation procedure described here for each of the frequency ranges. 


\section{CONCLUSIONS}

In summary, we have constructed a model and presented simulations of diffuse scattering due to magnons. The magnon scattering has a relative intensity of up to $10^{-4}$ of the phonon scattering intensity, meaning that an experimental detection of a magnon signal appears very demanding, although recent instrumental developments are likely to at least partially alleviate the challenges. Successful fingerprinting of magnons in an electron microscope would create a radical way of studying the fundamentals of magnetic ordering and spin wave excitations, e.g., in material systems used for spintronics and spin caloritronics, where spin currents are propagated by magnons [37].

\section{ACKNOWLEDGMENTS}

We acknowledge the support of Swedish Research Council, Carl Trygger's Foundation and eSSENCE. Simulations were enabled by resources provided by the Swedish National Infrastructure for Computing (SNIC) at NSC Centre partially funded by the Swedish Research Council through Grant Agreement No. 2018-05973. SuperSTEM is the National Research Facility for Advanced Electron Microscopy funded by the Engineering and Physical Sciences Research Council (EPSRC). We acknowledge financial support from the Engineering and Physical Sciences Research Council (EPSRC) via Grant No. EP/V048767/1 and Royal Society Grant No IES/R1/211016. J.C.I. acknowledges support of the Center for Nanophase Materials Sciences, which is a DOE Office of Science User Facility.

\section{APPENDIX A: PARALLELS BETWEEN THE EXCITATION MECHANISMS OF PHONONS AND MAGNONS}

The description of electron-phonon scattering in multislice calculations makes use of the frozen phonon method, which is based on the idea that electrons passing through a configuration of vibrating atoms can be treated by having the atoms frozen in place, due to the period of vibration of the atom being much shorter than the interaction time of the electron in the system. This approach has been shown to be formally exact under certain conditions [38], with van Dyck [22] showing that in the case of single inelastic scattering and independent atom vibrations, this approach is fully equivalent to a quantum-mechanical description based on the Yoshioka equations. Following the formalism presented in that paper, with adjustments reflecting the magnetic rather than vibrational nature of the scattering mechanism under consideration, an analogous frozen magnon method is formalized.

Following Edström et al. [18], the time-independent paraxial Pauli equation is

$$
\begin{aligned}
\frac{\partial \Psi}{\partial z}= & \frac{i m}{\hbar}\left(\hbar k+e A_{z}\right)^{-1}\left\{\frac{\hbar^{2} \nabla_{x y}^{2}}{2 m}+\frac{i e \hbar}{m} A_{x y} \cdot \nabla_{x y}\right. \\
& \left.-\frac{\hbar k e A_{z}}{m}-\frac{e \hbar}{2 m} \sigma \cdot B+e V\right\} \Psi,
\end{aligned}
$$

where $\Psi$ is a two-component wave function with a spin up and spin down part, $\nabla_{x y}$ is the in-plane gradient operator,
$V$ is the electrostatic potential, and $k$ and $m$ are de Broglie wavelength of electron and electron mass, both relativistically corrected [27]. To the first order in the magnetic vector potential $A$, which is a valid approximation for fast electrons where $\hbar k \gg e A_{z}$, this becomes

$$
\begin{aligned}
\frac{\partial \Psi}{\partial z} \approx & \frac{i m}{\hbar^{2} k}\left\{\frac{\hbar^{2} \nabla_{x y}^{2}}{2 m}\right\} \Psi \\
& +\left[\frac{i m}{\hbar^{2} k}\left\{e V+\frac{i e \hbar}{m} A_{x y} \cdot \nabla_{x y}-\frac{\hbar k e A_{z}}{m}\right\}\right. \\
& \left.-\frac{i m e A_{z}}{\hbar^{3} k^{2}}\left\{\frac{\hbar^{2} \nabla_{x y}^{2}}{2 m}+e V\right\}-\frac{i e}{2 \hbar k} \sigma \cdot B\right] \Psi .
\end{aligned}
$$

This expression is analogous to Eq. (1) in van Dyck's paper [22], with the scattering mechanism being magnetic fields and potentials. In the following equation in the paper, it is assumed that the potential is time dependent and can be broken up as $V(t)=\langle V\rangle+W(t)$, with \langle\rangle representing an average taken over time and $\langle W(t)\rangle=0$. In contrast to the potential resulting from a vibrating lattice, in the case of magnon scattering there are terms in $W(t)$ dependent on $\nabla_{x y}$. Here we invoke the approximation that $\Psi$ under the influence of magnetic scattering should not change to the degree that terms dependent on $\nabla_{x y}$ will substantially change from snapshot to snapshot, thereby allowing an average over time for the potential to be taken.

There are two additional assumptions made through the derivation of the frozen phonon method in the paper [22] in Eqs. (3)-(36), that must be noted. First is that the scattering interaction term $\left\langle W(z, t) W\left(z^{\prime}, t\right)\right\rangle$ is assumed to be zero for $z \neq z^{\prime}$. For phonons, this is justified in that, for small atomic displacements, the perturbation in the crystal potential $W(t)$ is sharply peaked at the atom centres. The same assumption can be made for magnetic moments, with magnetic fields peaking within $1 \AA$ of atomic centres. The second assumption made is that the atom motions are uncorrelated to avoid a nonlocal component in the optical potential, although the same assumption is made in the quantum mechanical derivation. (a)

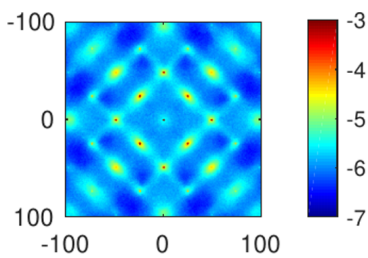

(c) -100

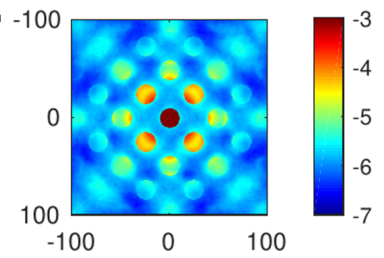

(b)

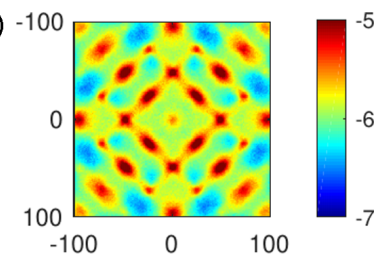

(d)

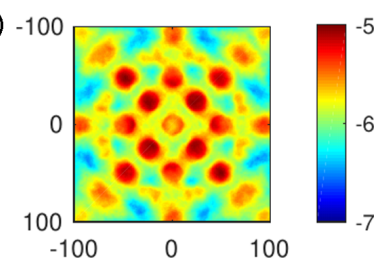

FIG. 4. Phonons at $30 \mathrm{kV}$ for a $5-\mathrm{nm}$ thick sample. The rest as in Fig. 1 of the main text. The convergence semiangle was set to $1 \mathrm{mrad}$ in (a) and (b), and to $10 \mathrm{mrad}$ in (c) and (d), respectively. Color bars refer to a decadic logarithm of intensity. 
(a) -100

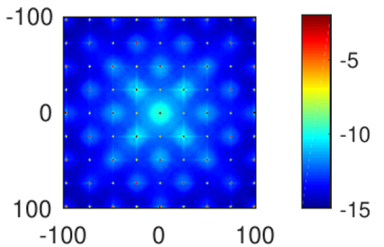

(c)

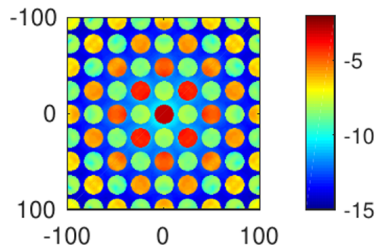

(b)

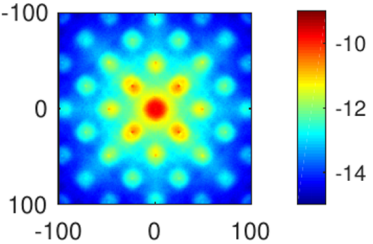

(d)

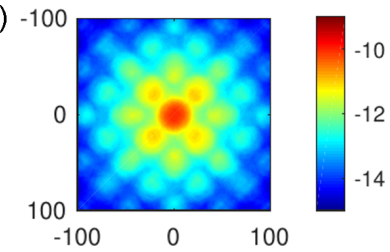

FIG. 5. Magnons at $30 \mathrm{kV}$ for a $5 \mathrm{~nm}$ thick sample. The rest as in Fig. 2 of the main text. The convergence semiangle was set to $1 \mathrm{mrad}$ in (a) and (b), and to $10 \mathrm{mrad}$ in (c) and (d), respectively. Color bars refer to a decadic logarithm of intensity.

Analogous to this is the assumption that magnetic moments are uncorrelated, i.e., an independent atom approximation.

Equations (37)-(77) in the van Dyck paper [22] outline the quantum mechanical treatment for describing electronphonon interactions in the multislice framework. In Eq. (37) of the paper, wave functions are expressed in a joint basis in the position vector of the electron $\mathbf{r}$ and that of atom $i \mathbf{r}_{i}$. For the frozen magnon method, assuming a fixed lattice, the wave function will instead be expressed as $\psi\left(\mathbf{r}, \mathbf{m}_{i}\right)$, where $\mathbf{m}_{i}$ is the vector describing the magnetic moment of the atom. Knowledge of the orientations of the magnetic moments of all atoms is sufficient to uniquely describe the magnetic vector fields $\mathbf{A}$ and $\mathbf{B}$ up to gauge invariance. Analogous to Eq. (38), eigenstates for a given magnetic moment configu-

[1] O. L. Krivanek, T. C. Lovejoy, N. Dellby, T. Aoki, R. W. Carpenter, P. Rez, E. Soignard, J. Zhu, P. E. Batson, M. J. Lagos et al., Nature (London) 514, 209 (2014).

[2] O. L. Krivanek, N. Dellby, J. A. Hachtel, J.-C. Idrobo, M. T. Hotz, B. Plotkin-Swing, N. J. Bacon, A. L. Bleloch, G. J. Corbin, M. V. Hoffman et al., Ultramicroscopy 203, 60 (2019).

[3] J. A. Hachtel, J. Huang, I. Popovs, S. Jansone-Popova, J. K. Keum, J. Jakowski, T. C. Lovejoy, N. Dellby, O. L. Krivanek, and J. C. Idrobo, Science 363, 525 (2019).

[4] F. S. Hage, D. M. Kepaptsoglou, Q. M. Ramasse, and L. J. Allen, Phys. Rev. Lett. 122, 016103 (2019).

[5] K. Venkatraman, B. D. A. Levin, K. March, P. Rez, and P. A. Crozier, Nat. Phys. 15, 1237 (2019).

[6] F. S. Hage, G. Radtke, D. M. Kepaptsoglou, M. Lazzeri, and Q. M. Ramasse, Science 367, 1124 (2020).

[7] X. Yan, C. Liu, C. A. Gadre, L. Gu, T. Aoki, T. C. Lovejoy, N. Dellby, O. L. Krivanek, D. G. Schlom, R. Wu, and X. Pan, Nature (London) 589, 65 (2021).

[8] O. Eriksson, A. Bergman, L. Bergqvist, and J. Hellsvik, Atomistic Spin Dynamics: Foundations and Applications (Oxford University Press, Oxford, 2017).

[9] S. K. Sinha, S. H. Liu, L. D. Muhlestein, and N. Wakabayashi, Phys. Rev. Lett. 23, 311 (1969).
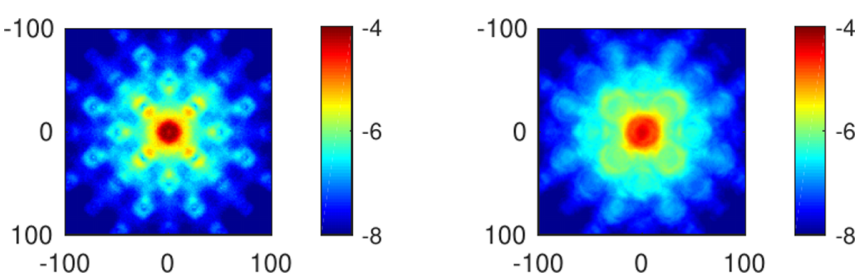

FIG. 6. Ratio of MDS and TDS at $1 \mathrm{mrad}$ (left) and $10 \mathrm{mrad}$ (right), respectively, both at $30 \mathrm{kV}$ for a 5-nm thick sample. Color bars refer to decadic logarithm of the ratio.

ration $\left|a_{n}\left(\mathbf{m}_{i}\right)\right\rangle$ exist and form a complete set for all functions over $\mathbf{m}_{i}$, so that

$$
\psi\left(\mathbf{r}, \mathbf{m}_{i}\right)=\sum_{m} \psi_{m}(\mathbf{r})\left|a_{m}\left(\mathbf{m}_{i}\right)\right\rangle .
$$

It is worth noting that the terms of the interaction part of the Hamiltonian, $\alpha_{n m}=\left\langle a_{n}\left(\mathbf{m}_{i}\right)\left|\mathcal{H}_{\text {int }}\right| a_{m}\left(\mathbf{m}_{i}\right)\right\rangle$, contain terms corresponding both to the electrostatic potential of the atoms and the magnetic vector fields as seen in Eq. (A1), analogous to the treatment of magnetism and magnons in the context of spin-polarized DFT [39]. Lastly, it is important to note that, while the approach above has been done for a fixed lattice for simplicity, none of the assumptions or formalism preclude the inclusion of lattice vibrations, which is especially important given the overlapping energy spectrum of these two quantized vibrations.

\section{APPENDIX B: ADDITIONAL FIGURES}

Additional Figs. 4-6 from simulations at $30 \mathrm{kV}$ acceleration voltage are presented here.

[10] P. S. Keatley, T. H. J. Loughran, E. Hendry, W. L. Barnes, R. J. Hicken, J. R. Childress, and J. A. Katine, Rev. Sci. Instrum. 88, 123708 (2017).

[11] T. Sebastian, K. Schultheiss, B. Obry, B. Hillebrands, and H. Schultheiss, Front. Phys. 3, 35 (2015).

[12] R. Vollmer, M. Etzkorn, P. Anil Kumar, H. Ibach, and J. Kirschner, J. Magn. Magn. Mater. 272-276, 2126 (2004).

[13] K. Zakeri, Y. Zhang, and J. Kirschner, J. Electron Spectrosc. Relat. Phenom. 189, 157 (2013).

[14] H. Ibach, F. C. Bocquet, J. Sforzini, S. Soubatch, and F. S. Tautz, Rev. Sci. Instrum. 88, 033903 (2017).

[15] A. Rother and K. Scheerschmidt, Ultramicroscopy 109, 154 (2009).

[16] J. C. Loudon, Phys. Rev. Lett. 109, 267204 (2012).

[17] A. Edström, A. Lubk, and J. Rusz, Phys. Rev. Lett. 116, 127203 (2016).

[18] A. Edström, A. Lubk, and J. Rusz, Phys. Rev. B 94, 174414 (2016).

[19] A. Edström, A. Lubk, and J. Rusz, Phys. Rev. B 99, 174428 (2019).

[20] B. Plotkin-Swing, G. J. Corbin, S. De Carlo, N. Dellby, C. Hoermann, M. V. Hoffman, T. C. Lovejoy, C. E. Meyer, A. Mittelberger, R. Pantelic, L. Piazza, and O. L. Krivanek, Ultramicroscopy 217, 113067 (2020). 
[21] B. D. Forbes, A. V. Martin, S. D. Findlay, A. J. D'Alfonso, and L. J. Allen, Phys. Rev. B 82, 104103 (2010).

[22] D. Van Dyck, Ultramicroscopy 109, 677 (2009).

[23] J. M. Cowley and A. F. Moodie, Acta Crystallogr. 10, 609 (1957).

[24] K. Lyon and J. Rusz, Acta Cryst. A 77, 509 (2021).

[25] The Uppsala atomistic spin dynamics code, UppASD, https: //github.com/UppASD/UppASD.

[26] H. Ebert, D. Ködderitzsch, and J. Minár, Rep. Prog. Phys. 74, 096501 (2011).

[27] E. J. Kirkland, Advanced Computing in Electron Microscopy (Springer, Boston, 2010).

[28] S. Plimpton, J. Comput. Phys. 117, 1 (1995).

[29] LAMMPS website, version 3Mar2020 stable, accessed: 202103-03 (2020), http://lammps.sandia.gov.

[30] M. I. Mendelev, S. Han, D. J. Srolovitz, G. J. Ackland, D. Y. Sun, and M. Asta, Philos. Mag. 83, 3977 (2003).
[31] F. S. Hage, Q. M. Ramasse, and L. J. Allen, Phys. Rev. B 102, 214111 (2020).

[32] P. M. Zeiger and J. Rusz, Phys. Rev. B 104, 094103 (2021).

[33] N. Dellby, T. Lovejoy, G. Corbin, N. Johnson, R. Hayner, M. Hoffman, P. Hrncrik, B. Plotkin-Swing, D. Taylor, and O. Krivanek, Microsc. Microanal. 26, 1804 (2020).

[34] N. Shibata, Y. Kohno, A. Nakamura, S. Morishita, T. Seki, A. Kumamoto, H. Sawada, T. Matsumoto, S. D. Findlay, and Y. Ikuhara, Nat. Commun. 10, 2308 (2019).

[35] P. M. Zeiger and J. Rusz, Phys. Rev. Lett. 124, 025501 (2020).

[36] P. M. Zeiger and J. Rusz, Phys. Rev. B 104, 104301 (2021).

[37] J. Sinova, S. O. Valenzuela, J. Wunderlich, C. H. Back, and T. Jungwirth, Rev. Mod. Phys. 87, 1213 (2015).

[38] Z. L. Wang, Acta Cryst. A 54, 460 (1998).

[39] N. Tancogne-Dejean, F. G. Eich, and A. Rubio, J. Chem. Theory Comput. 16, 1007 (2020). 